\title{
Minimal vertex covers of random trees
}

\author{
Stéphane Coulomb ${ }^{1}$ \\ Service de Physique Théorique de Saclay ${ }^{2}$ \\ CE Saclay, 91191 Gif sur Yvette, France
}

\begin{abstract}
We study minimal vertex covers of trees. Contrarily to the number $N_{v c}(A)$ of minimal vertex covers of the tree $A, \log N_{v c}(A)$ is a self-averaging quantity. We show that, for large sizes $n, \lim _{n \rightarrow+\infty}<$ $\log N_{v c}(A)>_{n} / n=0.1033252 \pm 10^{-7}$. The basic idea is, given a tree, to concentrate on its degenerate vertices, that is those vertices which belong to some minimal vertex cover but not to all of them. Deletion of the other vertices induces a forest of totally degenerate trees. We show that the problem reduces to the computation of the size distribution of this forest, which we perform analytically, and of the average $<\log N_{v c}>$ over totally degenerate trees of given size, which we perform numerically.
\end{abstract}

\section{Introduction}

The vertex-cover problem, as other combinatorial problems, is arousing growing interest in the fields of statistical physics and disordered systems. In particular, it helps to understand, and the machinery of optimization algorithms helps to solve, spin-glasses and random hamiltonian models (see [1] for a recent review of the problem, [2] for a critical analysis point of view). A possible question is : given a graph, what can be said about the size and number of its minimum vertex covers ? Another approach consists in answering this question on average, for a given statistical ensemble of graphs.

In this paper, we are concerned with average behaviour, and focus on the simple situation of trees. In this case, good algorithms are known, for

\footnotetext{
${ }^{1}$ Email: coulomb@spht.saclay.cea.fr

${ }^{2}$ Laboratoire de la Direction des Sciences de la Matière du Commisariat à l'Energie Atomique, URA2306 du CNRS
} 
instance based on the so-called b-colorings (see [3] and sect 2), to find the number $N_{v c}(A)$ or size of minimal vertex covers of a given tree $A$. In fact, if each tree of given size $n$ has the same probability, then the average number of vertex covers can also be retrieved analytically by means of these b-colorings. However, this is not a self-averaging quantity for large $n$, and it would be desirable to find a thermodynamically extensive quantity giving a somewhat more physical insight into the number of minimal configurations of a random tree.

We claim that $<\log N_{v c}(A)>_{n}$ is indeed self-averaging, and the reason is as follows. Suppose that we delete from $A$ all the vertices which are not degenerate (that is, those which belong either to all the minimal vertex covers of $A$ or to none of them). Then we obtain (see sect 3) a forest with the same number of minimal vertex covers as $A$ and whose vertices are all degenerate. Moreover, in this forest, the number of trees of given size scales thermodynamically with the size of $A$ (see sect 4), and the probability of appearance of a given tree depends only on its size. In other words, as far as we are concerned with the number of minimal vertex covers, picking at random a tree on $n \gg 1$ vertices amounts for each $i \geq 1$ to picking with uniform law $c_{i} n$ totally degenerate trees on $i$ vertices. And, in turn, it is expected that such a typical tree $A$ verifies $\log N_{v c}(A) \approx n \sum_{i} c_{i}<\log N_{v c}>_{i}^{R}$, where $<\log N_{v c}>_{i}^{R}$ is the average of $\log N_{v c}$ over totally degenerate trees on $i$ vertices.

The computation thus reduces to that of the scaling parameters $c_{i}$ for the size distribution, and to the evaluation of the average of $\log N_{v c}$ over totally degenerate trees with given size (see sect 5 ).

But let us begin with a remainder of some basic facts and the crucial theorem on b-colorings.

\section{Preliminary observations}

\subsection{Basic definitions}

A graph is a pair $A=(V, \mathcal{E})$ where $V$ is a set with $n \geq 1$ elements (written $|V|=n$ in the sequel) and $\mathcal{E}$ is a subset of $\{\{x, y\} \subset V ; x \neq y\} . V$ is the set of vertices of $A$ and $\mathcal{E}$ the set of edges of $A, n$ is the size of $A$, denoted by $|A|$. In this paper $A$ is called a labeled graph if $V$ consists of positive integers.

Given two distinct vertices $x, y$ of the graph $A=(V, \mathcal{E})$, a path from $x$ to $y$ in $A$ is a sequence $\left\{v_{0}, v_{1}\right\},\left\{v_{1}, v_{2}\right\}, \cdots,\left\{v_{p-1}, v_{p}\right\}$ of edges of $A$ such that $v_{0}=x, v_{p}=y$ and $v_{i} \neq v_{j}$ if $i \neq j$. A graph is called a tree if any two distinct vertices are connected by a unique path, and a forest if any two 
distinct vertices are connected by at most one path.

A rooted tree is a triple $(V, \mathcal{E}, r)$, such that $(V, \mathcal{E})$ is a tree and $r \in V$.

A vertex cover of the graph $A=(V, \mathcal{E})$ is a subset of $V$ containing at least one end of each edge of $A$. A vertex cover of $A$ is minimal if there does not exist any other vertex cover with less elements. In the sequel, the number of minimal vertex covers of $A$ is denoted $N_{v c}(A)$.

\subsection{Some useful results}

The exponential generating function of rooted trees It is defined as $T(x)=\sum_{A} \frac{x^{|A|}}{|A| !}$, where the sum runs over all rooted trees. Cayley's formula states that the number of rooted trees on $n$ vertices is $n^{n-1}$, hence $T(x)=\sum_{n \geq 1} \frac{n^{n-1}}{n !} x^{n}$ and this implies that $T(x)=x e^{T(x)}$ as can be deduced by a direct combinatorial argument relying on the recursive nature of rooted trees.

A theorem on minimal vertex covers of trees It has been shown in [3] that, for any tree $A=(V, \mathcal{E})$, there exists a unique triple $(B, \mathcal{R}, G) \subset$ $V \times \mathcal{E} \times V$, called the $b$-coloring of $A$, such that

- $B, G$ and the set of end-vertices of $\mathcal{R}$ form a partition of $V$.

- The edges in $\mathcal{R}$ are non-adjacent; the edges with one end-vertex in $G$ have the other end-vertex in $B$; each vertex in $B$ is connected to $G$ by at least two edges.

Moreover, the b-coloring of $A$ has the following connection with its minimal vertex covers : $B$ (resp. $G$ ) is the set of vertices contained in all (resp. none) of the minimal vertex covers of $A$. Consequently, any end-vertex of $\mathcal{R}$ is contained in some minimal vertex cover of $A$ but not in all of them : these vertices are called degenerate.

An additional result is that any minimal vertex cover of $A$ contains exactly one end-vertex of each edge in $\mathcal{R}$. Consequently, a vertex cover of $A$ is minimal if and only if it contains $|B|+|\mathcal{R}|$ vertices.

In the sequel, vertices in $B$ and $G$ and end-vertices of $\mathcal{R}$ will be called respectively brown, green and red vertices, while edges in $\mathcal{R}$ will be called red edges. A tree with no brown or green vertices is said to be red.

\section{Red forest of a tree}

Given a tree $A=(V, \mathcal{E})$ and a non-empty set $S \subset V$ of vertices, the forest induced by $A$ on $S$ is defined as $\left(S, \mathcal{E}^{\prime}\right)$, where $\mathcal{E}^{\prime}$ consists of those edges in 
$\mathcal{E}$ with both ends in $S$. If $A$ is a tree with b-coloring $(B, \mathcal{R}, G)$, and such that $\mathcal{R} \neq \emptyset$, define the red forest of $A$ to be the forest induced by $A$ on the set of red vertices. Denote $A_{1}=\left(V_{1}, \mathcal{E}_{1}\right), \cdots, A_{p}=\left(V_{p}, \mathcal{E}_{p}\right)$ the trees of that forest. Then it follows at once from the definitions that $A_{i}$ has b-coloring $\left(\emptyset, \mathcal{R} \cap \mathcal{E}_{i}, \emptyset\right)$, hence is red. But if $C$ is a minimal vertex cover of $A, C \cap V_{i}$ is a vertex cover of $A_{i}$. Since $C$ contains exactly one end of each red edge of $A, C \cap V_{i}$ contains $\left|\mathcal{R} \cap \mathcal{E}_{i}\right|$ vertices of $A_{i}$ : it is a minimal vertex cover of $A_{i}$. Now, given minimal vertex covers $C_{1}, \cdots, C_{p}$ of the $A_{i}$ 's, $B \cup C_{1} \cup \cdots \cup C_{p}$ is a vertex cover of $A$ (because an edge of $A$ either is an edge of some $A_{i}$ or has at least one end in $B$ ), which is minimal since it contains $|B|+|\mathcal{R}|$ vertices. It is in fact the only minimal vertex cover of $A$ which coincides with $C_{i}$ on each $A_{i}$, and this proves that :

$$
N_{v c}(A)=\prod_{i=1}^{p} N_{v c}\left(A_{i}\right) .
$$

Let us define the size distribution of a forest $F$ as the sequence $D=$ $\left(D_{i}\right)_{i \geq 1}$, where $D_{i}$ is the number of components of size $i$ in $F$. Given two forests $F_{1}, F_{2}$ of red trees, with same size distribution $D$, there is no difficulty in proving that the numbers of trees with red forests respectively $F_{1}$ and $F_{2}$ are equal. In other words the number of trees on $n$ vertices with given red forest $F$ depends on $F$ only via its size distribution $D$ : this number shall be denoted $\nu_{D}(n)$ in the sequel. Note that $\nu_{D}(n)=0$ if $D_{i} \neq 0$ for some $i>n$.

If we denote by $\lambda_{i}$ the sum over red trees $R$ on $i$ vertices of $\log N_{v c}(R)$, the preceding remarks allow to write our sum over trees of size $n$ as

$$
\sum_{A} \log N_{v c}(A)=\sum_{D} \nu_{D}(n)\left(D_{1} \lambda_{1}+D_{2} \lambda_{2}+\cdots+D_{n} \lambda_{n}\right) .
$$

We are thus led to the computation of the $\nu_{D}$ 's and $\lambda_{i}$ 's. Note already that a red tree has even size, whence $\nu_{D}(n)=0$ if $D_{2 i+1} \neq 0$ for some $i$. We now come to the analytic computation of $\nu_{D}(n)$.

\section{Size distribution}

Denote by $G, B, R$ respectively the exponential generating functions for the number of rooted trees with root of color green, brown and red. For instance, $G(x) \equiv \sum_{A} \frac{1}{|A| !} x^{|A|}$, where the sum runs over all rooted trees with green root. The following relations hold between these generating functions (see [3] for details and the combinatorial meaning of $U, Q$ )

$$
G=x e^{U}
$$




$$
\begin{aligned}
U & =x e^{B+R}\left(e^{G}-1\right) \\
B & =x e^{B+R}\left(e^{G}-1-G\right) \\
R & =x Q e^{B+R} \\
Q & =x e^{B+R}
\end{aligned}
$$

leading in particular to $B(x)=T(x)+T(-T(x))-T(-T(x))^{2}$. Now, let us look more closely at those trees with red root. The red forest of such a tree $A$ has exactly one component containing the root, and the size $s(A)$ of this component can be encoded in the following generating function, where the sum runs over rooted trees with red root

$$
R_{0}(x, y) \equiv \sum_{A} \frac{1}{|A| !} x^{|A|} y^{s(A)} .
$$

Since $R_{0}=x y Q_{0} e^{B+R_{0}}$ with $Q_{0}=x y e^{B+R_{0}}$, it follows that $R_{0}=T\left(2 x^{2} y^{2} e^{2 B(x)}\right) / 2$, and the total number of red components of size $2 p$ among labeled trees of size $n$ is

$$
\frac{1}{2 p} n ![x]_{n}[y]_{2 p} R_{0}(x, y)=\frac{(2 p)^{p-2}}{p !} n ![x]_{n-2 p} e^{2 p B(x)}
$$

A straightforward application of the saddle-point method then shows that, for large $n$, the average number of red components of size $2 p$ scales thermodynamically with $n: C_{2 p}(n) \sim c_{2 p} n$ and

$$
c_{2 p}=\frac{(2 p)^{p-1}}{p !} T^{\prime} T^{2 p-1} e^{-2 p T^{2}}\left(2 T^{2}-1\right)
$$

where, in the above formula, $T(x)$ and its derivative are taken at the saddlepoint $x=-1$. For large $p$, we get that $\frac{\log c_{2 p}}{p}$ tends to $\log \left(2 e T^{2} \exp \left(-2 T^{2}\right)\right) \approx$ -0.0844424236 , showing that $c_{2 p}$ decays exponentially.

Now, we make the "thermodynamic limit" assumption that the number of trees with given size in the red forest of some random large tree is a selfaveraging quantity. That is, we suppose that, for large $n$, the trees which contribute significantly to $<\log N_{v c}>_{n}$ have indeed $C_{2}$ trees of size $2, C_{4}$ trees of size $4, \cdots$. The distribution $\nu_{D}$ hence becomes irrelevant, since it concentrates on one particular value, and the average becomes

$$
\lim _{n \rightarrow+\infty}<\log N_{v c}>_{n} / n=\sum_{i} c_{2 i} \frac{\lambda_{2 i}}{N_{2 i}},
$$

where $N_{2 i}$ denotes the number of red trees of size $2 i$. 


\section{Minimal vertex covers of the red trees}

In this section, we compute analytically the number $N_{2 p}$ of red trees on $2 p$ vertices and give a numeric estimate of the $\lambda_{2 i}$ 's. In fact, one could deduce directly $R(x)$, whence the $N_{2 p}$ 's, from the set of equations on $B, G, R, Q, U$ stated in the preceding section. But we prefer to give a direct combinatorial derivation which, after slight adaptations, shall give also the total number of minimal vertex covers among red trees of size $2 p$.

\section{$5.1 \quad$ Overview}

As was already emphasized, a red tree $A$ has an even number of vertices, say $2 p$, and we associate to $A$ its shrinked tree $\tilde{A}$ as follows

- The vertices of $\tilde{A}$ are the red edges of $A$, so $\tilde{A}$ has size $p$;

- Two vertices of $\tilde{A}$ are connected in $\tilde{A}$ if and only if the corresponding two red edges of $A$ are connected by some other edge in $A$.

This procedure is uniquely defined and, if the set of vertices of $A$ is $V$, that of its shrinked tree is a partition of $V$ into sets of 2 elements. Such a partition will be called a pairing of $V$ : note that it consists of the red edges of $A$.

Conversely, let $V$ be a set $(|V|=2 p)$. There are $\frac{(2 p) !}{2^{p} p !}$ pairings of $V$, and $p^{p-2}$ trees with set of vertices equal to one of these pairings. Given such a tree $B$, the number of red trees on $V$ with shrinked tree $B$ is $4^{p-1}$, because each of the $p-1$ edges of $B$ leaves 4 possibilities for the corresponding edge of the red tree. Hence, the number of red trees on $2 p$ vertices is

$$
N_{2 p}=\frac{(2 p) !}{p !}(2 p)^{p-2}
$$

so the number $2 p N_{2 p}$ of rooted trees has exponential generating function $R(x)=T\left(2 x^{2}\right) / 2$.

Let us now enumerate the total number of minimal vertex covers among the red trees of size $2 p$. Consider a minimal vertex cover on a labeled tree $A$ of size $2 p$. To encode this vertex cover, add an arrow at each covered end of each black edge (that is, each edge which is not red). By definition of vertex covers a black edge is either oriented (one arrow) or bi-oriented (two arrows).

Now, we apply the shrinking procedure as defined above, but we keep track of the orientations : this leads to a tree on $p$ vertices, each edge being either oriented or bi-oriented.

Again this procedure is uniquely defined. If $V$ is a set on $2 p$ vertices, the number of trees with set of vertices a pairing of $V$ and with edges either 
oriented or bi-oriented is $\frac{(2 p) !}{2^{p} p !} p^{p-2} 3^{p-1}$. Given one such tree $B$, the number of covered red trees $A$ with shrinked tree $B$ is $2^{p}$. Indeed, each of the $p$ vertices of $B$ corresponds to a red edge of $A$, which may be covered in two ways. Once this choice has been made, the way the black edges connect the red edges with each other is completely constrained by their (bi-)orientation.

Hence, the total number of minimal vertex covers over red trees of size $2 p$ is $3 \frac{(2 p) !}{p !}(3 p)^{p-2}$, and the average number of minimal vertex covers among red trees on $2 p$ vertices is $\left\langle N_{v c}>_{2 p}^{R}=2(3 / 2)^{p-1}\right.$.

\subsection{Theoretical viewpoint}

Both for theoretical understanding and for numerical purpose, it proves useful to focus on rooted trees, and we denote by $n_{+}(A)$ (resp. $\left.n_{-}(A)\right)$ the number of minimal vertex covers which contain (resp. do not contain) the root of the rooted red tree $A$.

A red tree with root $r$ may be seen recursively as an edge $\left\{r, r^{\prime}\right\}$, with both ends connected to the root of arbitrarily many red rooted trees. And it is clear (see [3] for details) that a set $S$ of vertices of $A$ is a minimal vertex cover of $A$ if and only if : (i) it induces a minimal vertex cover on each of these attached subtrees (ii) exactly one end of $\left\{r, r^{\prime}\right\}$ is not in $S$ (iii) the edges incident at this vertex have the other end in $S$. Consequently, denoting by $A_{i}$ the red trees attached to $r$ and by $A_{j}^{\prime}$ those attached to $r^{\prime}$ :

$$
\begin{aligned}
& n_{+}(A)=\prod\left(n_{+}\left(A_{i}\right)+n_{-}\left(A_{i}\right)\right) \prod n_{+}\left(A_{j}^{\prime}\right) \\
& n_{-}(A)=\prod n_{+}\left(A_{i}\right) \prod\left(n_{+}\left(A_{j}^{\prime}\right)+n_{-}\left(A_{j}^{\prime}\right)\right)
\end{aligned}
$$

Now, let us have a closer look at the generating function for rooted red trees $R(x)=T\left(2 x^{2}\right) / 2$. As follows from the equation for $T, R$ should be such that $R(x)=x^{2} e^{2 R(x)}$. Combinatorially, this means that the number of rooted red trees on $2 p$ vertices is

$$
(2 p) !\left[x^{2 p-2}\right]\left(\sum_{k \geq 0} \frac{1}{k !}\left[\sum_{A} \frac{x^{|A|}}{|A| !}\right]^{k}\right)\left(\sum_{k^{\prime} \geq 0} \frac{1}{k^{\prime} !}\left[\sum_{A} \frac{x^{|A|}}{|A| !}\right]^{k^{\prime}}\right)
$$

where $A$ ranges over rooted red trees. But building a rooted tree on $n$ vertices amounts to choosing (i) the root $r$ and the vertex $r^{\prime}$ with whom $r$ shares its red edges $\left(2 p(2 p-1)\right.$ ways) (ii) the numbers $k$ and $k^{\prime}$ of rooted trees attached respectively to those vertices (iii) those trees themselves $A_{1}, \cdots, A_{k}$ and $A_{1}^{\prime}, \cdots, A_{k^{\prime}}^{\prime}$, in such a way that their total number of vertices is $2 p-$ 2 (iv) finally, a relabeling of those trees which exhausts the labels $\neq r, r^{\prime}$ 
$\left((2 p-2) ! /\left(\prod\left|A_{i}\right| ! \prod\left|A_{j}^{\prime}\right| !\right)\right.$ ways $)$. Each term of the expansion of $\left[\sum_{A} \frac{x^{|A|} \mid}{|A| !}\right]^{k}$ corresponds to a particular ordered choice in (iii), and the $1 / k$ ! factor just gets rid of this ordering. This is true also for the primed term, hence the combinatorial meaning of the equation for $R$ is clear and we now apply it to our vertex covers problem.

The set $S$ of functions $\mathbb{N}^{2} \rightarrow \mathbb{R}$ is a vector space. If $\phi$ is such a function, and $\phi(a, b)=x_{a b}, a, b \in \mathbb{N}$, we write $\phi=\sum_{a, b} x_{a b}(a, b)$. If $\psi=\sum_{a, b} x_{a b}^{\prime}(a, b)$ is another function, let their product be $\phi * \psi=\sum_{a, b, a^{\prime}, b^{\prime}} x_{a b} x_{a^{\prime} b^{\prime}}^{\prime}\left(a a^{\prime}, b b^{\prime}\right)$. $S$ is then an algebra, generated by the $(a, b), a, b \in \mathbb{N}$. Let $\sigma$ be the (algebra) morphism such that $\sigma(a, b)=(b, a)$ for all $a, b$ and $\rho$ the (vector space) morphism such that $\rho(a, b)=(a+b, a)$. Then eqs. (23) rewrite $\left(n_{+}(A), n_{-}(A)\right)=\prod \rho\left(n_{+}\left(A_{i}\right), n_{-}\left(A_{i}\right)\right) * \prod \sigma \rho\left(n_{+}\left(A_{j}^{\prime}\right), n_{-}\left(A_{j}^{\prime}\right)\right)$. Hence, our remarks on the combinatorial meaning of eq.(4) show that the formal power series $R_{+-}(x) \equiv \sum_{A}\left(n_{+}(A), n_{-}(A)\right) \frac{x^{|A|}}{|A| !}$ obeys the equation

$$
R_{+-}(x)=x^{2} e^{\rho R_{+-}(x)+\sigma \rho R_{+-}(x)} .
$$

Of course, in this equation, the exponential is defined by its power series, the product being as defined above.

Let $f_{l m}$ be the (algebra) morphism such that $f_{l m}(a, b)=a^{l} b^{m}$ for all $a, b$. Then $f R_{+-}(x)=x^{2} e^{f \rho R_{+-}(x)+f \sigma \rho R_{+-}(x)}$, so we have the following generating functions for rooted trees:

$$
\begin{aligned}
R_{l m}(x) & \equiv \sum_{A} n_{+}(A)^{l} n_{-}(A)^{m} \frac{x^{|A|}}{|A| !} \\
& =x^{2} \exp \left(\sum_{k=0}^{l}\left(\begin{array}{l}
l \\
k
\end{array}\right) R_{k, l+m-k}+\sum_{k=0}^{m}\left(\begin{array}{c}
m \\
k
\end{array}\right) R_{k, l+m-k}\right)
\end{aligned}
$$

For the first two values of $n$, the resulting system of equations is easily solved. For instance :

For $l=m=0: \quad R_{0,0}(x)=x^{2} e^{2 R_{0,0}(x)}$, so $R_{0,0}(x)=T\left(2 x^{2}\right) / 2$ as expected.

For $l=1, m=0$ or $l=0, m=1: R_{1,0}(x)=R_{1,0}(x)=x^{2} e^{3 R_{1,0}(x)}$, so $R_{1,0}(x)=T\left(3 x^{2}\right) / 3$, again in agreement with the formula above.

And this seems to be the largest value of $n$ for which the exact solution functions are retrievable. In the case where $l+m=2$, the system reduces to an implicit expression for $R_{2,0}: R_{2,0}=x^{2} \exp \left(3 R_{2,0}+2 R_{2,0} e^{-R_{2,0}}\right)$, still allowing asymptotic computations. However, we have not found a systematic 


\begin{tabular}{c|ccc}
$k$ & 1 & 2 & 3 \\
\hline$M_{k}$ & 0.20273 & 0.41576 & 0.63658
\end{tabular}

Table 1: Moments $M_{k}=\lim _{p \rightarrow+\infty} \frac{1}{2 p} \log <N_{v c}^{k}>_{2 p}^{R}$ of the number of minimal vertex covers of red trees, as obtained from eq.(5).

treatment for the study of eq.(5) which would have been a possible starting point for the replica method.

We now come to the numerical evaluation of the $\lambda_{2 p}$ 's.

\subsection{Numerical computations}

Given a red tree $A$ on $2 p$ vertices, one can choose any of its vertices as a root and apply recursively equations (23) to compute $n_{+}(A), n_{-}(A)$ in $O(p)$ time. However, the number of such trees increases exponentially with $p$, and systematic enumeration soon becomes a challenge.

For small trees $(p \leq 16)$, we compute the exact distribution of the number of minimal vertex covers. The algorithm is based on an exhaustive recursive enumeration of rooted trees [4], followed by systematic unshrinking.

For larger trees, we proceed as follows. The number of red trees with given shrinked tree $A$ depends only on $|A|$, and every red tree on $2 p$ vertices has a unique shrinked tree, which is of size $p$. Hence, to pick randomly a red tree on $2 p$ vertices with uniform law, it suffices to : (i) Pick randomly a tree $A$ on $p$ vertices, with uniform law (this is conveniently done by means of the Prüfer bijection between those trees and sequences of $\{1, \cdots, p\}^{\{1, \cdots, p-2\}}$ ) (ii) Choose, again with uniform probability, one of the red trees with shrinked tree $A$.

The number of samples picked for each size was chosen so as to ensure a precision of $10^{-7}$ on $<\log N_{v c}>/ n$. From the fact that $<\log N_{v c}>_{n}$ $/ n=\sum_{i} c_{2 i} \lambda_{2 i} / N_{2 i}$, it follows that an error $\delta_{2 i}$ on $\frac{1}{2 i} \frac{\lambda_{2 i}}{N_{2 i}}$ leads to a maximum error $\sum_{i} c_{2 i} 2 i \delta_{2 i}$ on $<\log N_{v c}>_{n} / n$. From eq.(11) we see that $c_{2 i}$ decays exponentially fast with $i$ : in practice, we took $810^{9}$ samples for each size $17 \leq p \leq 45$ and $1.510^{8}$ samples for sizes $46 \leq p \leq 189$. And this leads to

$$
\lim _{n \rightarrow+\infty}<\log N_{v c}(A)>_{n} / n=\sum_{p>0} c_{2 p}<\log N_{v c}>_{2 p}^{R}=0.1033252 \pm 10^{-7}
$$

Those numerical simulations also give evidence that, for red trees of large size $2 p$, the random variable $X_{p}=\left(\log N_{v c}\right) /(2 p)$ is self-averaging. Indeed, for each of the sizes considered in the previous paragraph, it is possible to get the approximate distribution of $X_{p}$, and it appears that $\left(X_{p}-<X_{p}>\right) \sqrt{p}$ 
approaches a fixed gaussian distribution for large $p$. Numerically, we find $\lim _{p \rightarrow+\infty}<X_{p}>=\lim _{p \rightarrow+\infty}<\log N_{v c}>_{2 p}^{R} / 2 p=0.1963 \pm 10^{-4}$, to be compared with the first few moments of table [1. In fact, approximating the first few $M_{k}$ 's by a rational function leads to estimate $\lim _{p \rightarrow \infty}<X_{p}>=$ $\left.\frac{d M_{k}}{d k}\right|_{k=0} \approx 0.196$, a result remarkably close to the expected limit. Good understanding of this self-averaging feature would certainly be a crucial issue in the exact derivation of $\lim _{p \rightarrow+\infty}<X_{p}>$, and presumably also of the corresponding limit for general trees.

I am very grateful to Michel Bauer for interesting remarks and discussions.

\section{References}

[1] A. K. Hartmann, M. Weigt, Statistical mechanics of the vertex-cover problem, J. Phys. A: Math. Gen. 36 (2003) 11069-11093.

[2] M. Bauer, O. Golinelli, Core percolation in random graphs : a critical phenomena analysis, Eur. Phys. J. B 24 (2001), 339-352, cond-mat/0102011.

[3] S. Coulomb, M. Bauer, On vertex covers, matchings and random trees, cond-mat/0407456

[4] G. Li, F. Ruskey, The Advantages of Forward Thinking in Generating Rooted and Free Trees, SODA 1999: 939-940. 